\title{
Activin A regulates proliferation, invasion and migration in osteosarcoma cells
}

\author{
JIANWEI ZHU ${ }^{1}$, FAN LIU ${ }^{1}$, QUANMING WU ${ }^{1}$ and XIANCHENG LIU ${ }^{2}$
}

Departments of ${ }^{1}$ Orthopedics and ${ }^{2}$ Oncology, Affiliated Hospital of Nantong University, Nantong, Jiangsu 226001, P.R. China

Received April 13, 2014; Accepted January 2, 2015

DOI: $10.3892 / \mathrm{mmr} .2015 .3284$

\begin{abstract}
Activin A is a member of the TGF- $\beta$ superfamily. Previous studies have demonstrated that activin A exhibited pluripotent effects in several tumours. However, the roles of activin A signaling in osteosarcoma pathogenesis have not been previously investigated. Therefore, the present study aimed to investigate the effects of activin A on osteosarcoma cell proliferation, invasion and migration. Firstly, the expression of activin A in osteosarcoma cell lines (MG63, SaOS-2 and U2OS) and a human osteoblastic cell line (hFOB1.19) was detected using reverse transcription quantitative polymerase chain reaction and western blotting. Activin A was upregulated in osteosarcoma cell lines compared with hFOB1.19 cells. To investigate the effects of activin A on osteosarcoma cell proliferation, invasion and migration, MG63 cells were generated in which activin A was either overexpressed or depleted. MTT staining, propidium iodide staining and a Transwell assay were used to analyze the cell cycle, proliferation, invasion and migration of MG63 cells, respectively. The results of the present study revealed that the abilities of proliferation, invasion and migration were suppressed in MG63 cells in which activin A was depleted, while they were enhanced in activin A-overexpressing cells. In conclusion, the results of the present study suggested that activin A may facilitate proliferation, invasion and migration of osteosarcoma cells, and it may therefore be a potential target for the treatment of osteosarcoma.
\end{abstract}

\section{Introduction}

Osteosarcoma (OS) is a rare, highly malignant tumor of the bone. It is the most common primary bone malignancy in childhood and adolescence (1-3). OS is primarily a malignant neoplasm of the long bones, with the greatest predilection for

Correspondence to: Dr Jianwei Zhu, Department of Orthopedics, Affiliated Hospital of Nantong University, 20 Xishi Road, Nantong, Jiangsu 226001, P.R. China

E-mail: zhujianwei_nt@163.com

Key words: osteosarcoma, activin A, proliferation, invasion, migration the metaphyses of the distal femur and proximal tibia (4). OS is a highly aggressive tumor that metastasizes primarily to the lung (5). Metastasis is not only a sign of deterioration but also the major cause of treatment failure and mortality (6). The prognosis is poor due to lack of effective treatment methods (7). Therefore, innovative approaches that target the invasion and metastasis of osteosarcoma are urgently required. To date, the molecular mechanisms behind osteosarcoma development and metastasis have remained elusive. Therefore, broadening our understanding of the pathogenesis and biology of metastatic osteosarcoma is a key factor for improving treatment results and identifying potential therapeutic targets (2).

Activin A, belonging to the TGF- $\beta$ protein superfamily, interacts with two structurally similar serine/threonine kinase receptors and initiates downstream signaling via Smads to regulate gene expression (8). Activin is a pleiotropic cytokine with broad tissue distributions. Activin was initially described as a protein, which induces the release of follicle stimulating hormone from the pituitary gland. In recent years, activin has exhibited various effects on multiple physiological and pathological processes, including inflammation, metabolism, homeostasis, repair, cytoprotection, immune responses and endocrine function $(9,10)$. Recent studies have demonstrated that activin has an important role in cell proliferation, differentiation, apoptosis and carcinogenesis $(8,11,12)$.

Activins are homo- or heterodimers composed of four different $\beta$ subunits termed $\beta \mathrm{A}, \beta \mathrm{B}, \beta \mathrm{C}$ and $\beta \mathrm{E}$, respectively. Activin $\mathrm{A}$, the dimer of two $\beta \mathrm{A}$ subunits, is critically involved in the regulation of cell growth and apoptosis (13). Activin A is a multi-functional cytokine. Matsuo et al (14) demonstrated that activin A had an antiproliferative effect on thyroid papillary carcinoma cells and had a pivotal effect on the control of thyroid tumorigenesis. Kaneda et al (15) revealed that activin A inhibited vascular endothelial cell growth and suppressed tumor angiogenesis in gastric cancer. Activin A exhibited an inhibitory role in the proliferation of breast cancer cells through the activation of Smads (16). Activin A is a potent inhibitor of proliferation of certain epithelial ovarian cancer cell lines (17). Activin A normally inhibits cancer development and progression; however, cancer cell growth in high-grade prostate cancer is not inhibited by activin A (18). Recently, activin A has been revealed to be overexpressed in various types of cancer (19). Several studies have revealed that activin A may enhance tumor formation and progression through its effect on the tumor microenvironment (10). 
Hoda et al (20) revealed that activin A was overexpressed in malignant pleural mesothelioma (MPM) cells and contributed to the malignant phenotype of MPM cells via regulation of cyclin D. The elevated levels of activin A are responsible for the development of gonadal tumors and a cachexia-like weight loss syndrome (21). The overexpression of activin A was also correlated with positive node stage, poor histological differentiation and perineural invasion. Yoshinaga et al (22) demonstrated that activin A enhanced matrix metalloproteinase (MMP)-7 activity via the transcription factor activator protein 1 in an oesophageal squamous cell carcinoma cell line. Suppression of activin A in OC3 oral carcinoma cells using small interfering (si)RNA may attenuate cell proliferation, migration and invasiveness (18). Inhibition of activin A action is a promising strategy for the treatment of the types of cancer overexpressing this factor (23). Advanced myeloma is associated with high circulating levels of activin A, supporting the theory for the use of activin A antagonists in myeloma, such as sotatercept (24). Despite its pluripotent effects, the roles of activin A signaling in osteosarcoma pathogenesis remain to be elucidated. Therefore, the present study examined activin A expression in osteosarcoma cell lines (MG63, SaOS-2 and $\mathrm{U} 2 \mathrm{OS}$ ) and a human osteoblastic cell line (hFOB1.19) by reverse transcription quantitative polymerase chain reaction (RT-qPCR) and western blot analysis and observed changes in the viability, cell cycle as well as invasion and migration ability of MG-63 cells following up- or downregulation of activin A expression in human osteosarcoma MG-63 cells. The present study provided information which may aid in the development of prognosis prediction tools and targeted therapy for osteosarcoma.

\section{Materials and methods}

Reagents. Cell culture reagents were purchased from Gibco-BRL (Gaithersburg, MD, USA). Osteosarcoma cell lines (MG63, SaOS-2, U2OS) and a human osteoblastic cell line (hFOB1.19) were attained from the American Type Culture Collection (Manassas, VA, USA). Lipofectamine ${ }^{\mathrm{TM}} 2000$, pcDNA3.1vector and pGEM-T vector were from Invitrogen (Carlsbad, CA, USA). Restriction endonucleases HindIII and BamHI were purchased from Promega (Madison, WI, USA). T4 DNA Ligation agent was also from Promega. Taq DNA polymerase was purchased from Fermentas (Vilnius, Lithuania). The activin A siRNA was purchased from RiboBio Co. (Guangzhou, China). Protein extraction buffer and propidium iodide (PI) were purchased from Sigma-Aldrich (St. Louis, MO, USA). The enhanced chemiluminescence kit was purchased from Pierce (Rockford, IL, USA). Matrigel was purchased from Collaborative Research, Inc. (Bedford, MA, USA). The Transwell invasion chamber was purchased from Costar (Cambridge, MA, USA). The rabbit anti-activin A polyclonal antibody was purchased from Biorbyt (San Francisco, CA, USA). The rabbit anti-MMP-9 polyclonal antibody was purchased from Abnova (Taipei, Taiwan). The rabbit anti- $\beta$-actin, rabbit anti-cyclin D1 and rabbit anti-MMP-2 polyclonal antibodies were purchased from Abbiotec (San Diego, CA, USA). The horseradish peroxidase-conjugated goat anti-rabbit immunoglobulin ( $\mathrm{Ig}) \mathrm{G}$ was purchased from Invitrogen Life Technologies (Carlsbad, CA, USA).
Cell culture. Osteosarcoma cell lines (MG63, SaOS-2 and U2OS) were maintained in RPMI 1640 (Gibco-BRL) supplemented with $10 \%$ fetal bovine serum (FBS; Gibco-BRL) and $1 \%$ penicillin/streptomycin (Gibco-BRL) at $37^{\circ} \mathrm{C}$. The human osteoblast cell line hFOB1.19 was maintained in Dulbecco's modified Eagle's medium (DMEM):Ham's F-12 (Gibco-BRL) containing $10 \%$ FBS and geneticin $(400 \mu \mathrm{g} / \mathrm{ml}$; Gibco-BRL) at $34^{\circ} \mathrm{C}$ in a humidified $5 \% \mathrm{CO}_{2}$ incubator.

$R T-q P C R$. Total RNA was extracted from cells according to the manufacturer's instructions. RNA was reverse-transcribed and cDNA was synthesized using an iScript cDNA synthesis kit (Bio-Rad, Hercules, CA, USA). cDNA was stored at $-80^{\circ} \mathrm{C}$ until further use. All primers were purchased from Shanghai Generay Biotech Co., Ltd. (Shanghai, China). The primer sequences used were as follows: Activin A forward, 5'-ATAGCCCCTTTGCCAACCTC-3' and reverse, 5'-AGCACCTTAACGAAATGTAACTTGG-3'; and $\beta$-actin forward, 5'-GGCGGCCAACGCCAAAACTC-3' and reverse, 5'-GCCTCCGCCCGGTTCAAACA-3'. The qPCR reactions were performed using the iTaq Fast SYBR Green Supermix (Bio-Rad) according to the manufacturer's instructions. The cycle threshold $(\mathrm{Ct})$ values were determined and the relative mRNA expression was calculated using the $2^{-\Delta \Delta C t}$ method (25).

Western blot analysis. Total protein was extracted from cells using radioimmunoprecipitation buffer and quantified through a bicinchoninic acid assay kit (Sigma-Aldrich). Subsequently, the total proteins were separated using 12\% SDS-PAGE (Bio-Rad) and transferred onto polyvinylidene difluoride (PVDF) membranes (Amresco LLC, Solon, OH, USA). The PVDF membranes were blocked with 5\% milk/TBS-0.1\%-Tween (TBST; Bio-Rad) for $1 \mathrm{~h}$ at room temperature and probed with the appropriate primary antibody $(1: 1,000)$ at $4^{\circ} \mathrm{C}$ overnight. Subsequently, the PVDF membranes were washed in TBST for $3 \times 5$ min and probed with the corresponding secondary antibody for $2 \mathrm{~h}$ at room temperature. Following washing with TBST, autoradiography was conducted with enhanced chemiluminescence reagents. The relative expression of activin A was evaluated with the grey value ratio of activin A content to $\beta$-actin content (activin A/ $\beta$-actin).

Cell transfection. MG63 cells were transfected with plasmid containing pcDNA3.1-activin A (Invitrogen Life Technologies) or with activin A siRNA (RiboBio Co.) and Lipofectamine ${ }^{\mathrm{TM}} 2000$ (Invitrogen Life Technologies), used as the overexpression group and knockdown group, respectively. MG63 cells without any treatment were used as the control group. Transient transfection was performed using a high performance transfection reagent (Ribo FECT $^{\mathrm{TM}}$ CP Transfection kit; RiboBio Co.). Each stable transfectant clone was screened for overexpression or knockdown of activin A by RT-qPCR and western blot analysis.

MTT assay. MG63 cells were cultured on 96-well plates. MG63 cells in each well were incubated with $10 \mu 1 \mathrm{MTT}$ ( $5 \mathrm{mg} / \mathrm{ml}$; Beyotime, Haimen, China) for $4 \mathrm{~h}$. The formazan crystals formed from MTT by the living cells were dissolved in lysis buffer (Sigma-Aldrich). The purple solution of the formazan 
was detected using an OSE-260 spectrophotometer (Tiangen Biotech Co, Ltd, Beijing, China) to measure absorbance (A) at $570 \mathrm{~nm}$, and a $690-\mathrm{nm}$ measurement was used as a reference. The relative cell proliferation (\%) was calculated by the following equation: Relative proliferation rate $(\%)=$ [study $\operatorname{group}\left(\mathrm{A}_{570 \mathrm{~nm}}-\mathrm{A}_{690 \mathrm{~nm}}\right) /$ control group $\left.\left(\mathrm{A}_{570 \mathrm{~nm}}-\mathrm{A}_{690 \mathrm{~nm}}\right)\right] \times 100 \%$, as described in a previous study (26) and the experiment was performed in triplicate.

Cell cycle assay. MG63 cells were cultured in serum-free medium for $24 \mathrm{~h}$ for synchronization and then cultured in complete medium for $24 \mathrm{~h}$. Subsequently, the MG63 cells were detached with trypsin, washed and fixed in $70 \%$ cold ethanol overnight at $-20^{\circ} \mathrm{C}$. The fixed MG63 cells were washed with phosphate-buffered saline (Beyotime) and then treated with $1 \mathrm{mg} / \mathrm{ml} \mathrm{RNAse} \mathrm{(Beyotime)} \mathrm{for} 30 \mathrm{~min}$ at $37^{\circ} \mathrm{C}$. Subsequently, the MG63 cells were incubated with $\mathrm{PI}$ at a final concentration of $100 \mu \mathrm{g} / \mathrm{ml}$ at room temperature for $30 \mathrm{~min}$. The cell cycle was evaluated through flow cytometry (BD FACSCalibur ${ }^{\mathrm{TM}}$; BD Biosciences, San Jose, CA, USA) and the experiment was performed in triplicate.

Cell invasion assay. The Transwell invasion chamber was washed and then $50 \mu \mathrm{l}$ Matrigel $(1 \mathrm{mg} / \mathrm{ml})$ was added to evenly cover the Transwell inserts with $8-\mu \mathrm{m}$ pores to create the Matrigel membrane. The Transwell invasion chamber was divided into the outer chamber and the inner chamber by the Matrigel membrane. For invasion assays, MG63 cells $\left(4 \times 10^{5}\right)$ were serum-starved overnight and seeded in starvation medium on the outer chamber. The inner chamber contained 10\% FBS in RPMI 1640 medium. After $48 \mathrm{~h}$ incubation, MG63 cells from the outer chamber were removed from the surface of the matrigel membrane using a cotton swab. MG63 cells that had invaded into the lower compartment and attached to the lower surface of the filter were stained with Hoechst 33258 for $10 \mathrm{~min}$. Images of the invading cells were captured using an inverted microscope (XDS-1B; Wuzhou New Found Instrument Co., Ltd, Guangxi, China) and total cell numbers were counted and quantified.

Cell migration assay. The Transwell invasion chamber was washed with serum-free medium. The chamber was divided into upper and bottom chambers by the Transwell insert. For migration assays, MG63 cells $\left(4 \times 10^{5}\right)$ were serum-starved overnight and seeded in starvation medium on the upper chamber. The bottom chamber contained 10\% FBS in RPMI 1640 medium, which acted as a chemoattractant. After $48 \mathrm{~h}$ incubation, cells on the upper surface of the chamber were wiped off with cotton swabs. Cells in the Transwell inserts and on the lower surface of the inserts were washed into the bottom chamber with medium until no cells were on the inserts. Cells in the bottom chamber were incubated with MTT. The formazan crystals formed from MTT by the living cells were dissolved in lysis buffer. The relative number of cells was calculated from the optical density (OD) value using an OSE-260 spectrophotometer.

Statistical analysis. Values are presented as the mean \pm standard deviation of three individual experiments. The SPSS 17.0 statistical software (International Business Machines,
A

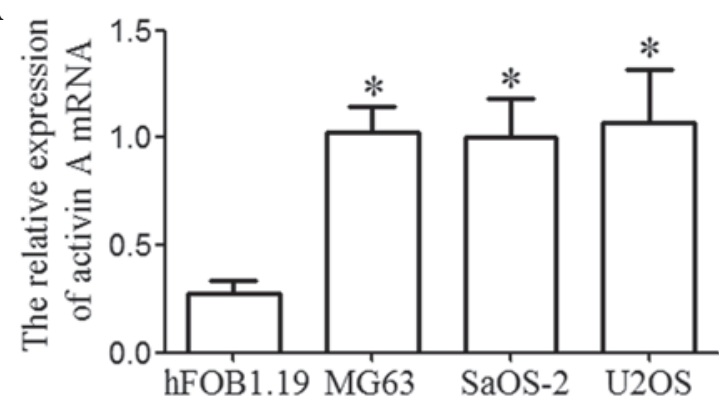

B

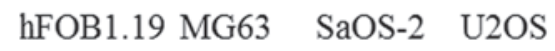

$\operatorname{activin} \mathrm{A}$

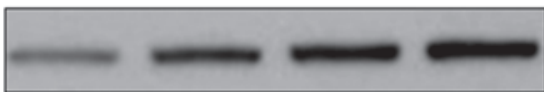

$\beta$-actin

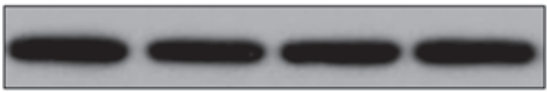

C

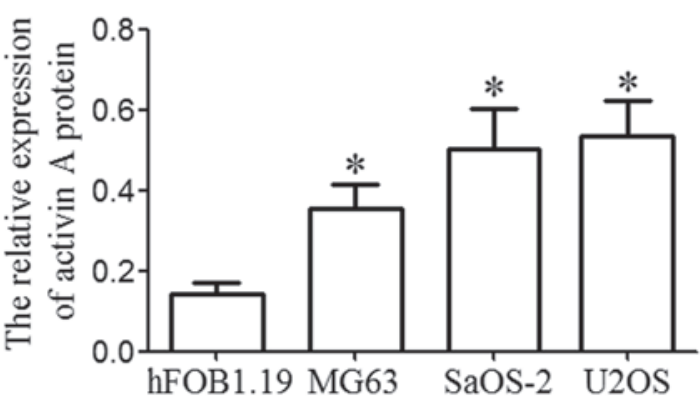

Figure 1. Expression of activin A in osteosarcoma cell lines. (A) Relative expression of activin A mRNA in osteosarcoma cell lines. (B) Western blot showing expression of activin A protein in osteosarcoma cell lines. (C) Relative expression of activin A protein in osteosarcoma cell lines, Values are presented as the mean \pm standard deviation of three individual experiments. ${ }^{*} \mathrm{P}<0.05$, compared with hFOB1.19.

Armonk, NY, USA) was used to analyze the quantitative data by one-way analysis of variance. $\mathrm{P}<0.05$ was considered to indicate a statistically significant difference.

\section{Results}

Activin A is overexpressed in osteosarcoma cell lines. In order to address the expression of activin A in osteosarcoma cells, activin A mRNA was detected by RT-qPCR and western blotting in three osteosarcoma cell lines (MG63, SaOS-2 and U2OS) and a human osteoblastic cell line (hFOB1.19). The expression levels of activin A mRNA and protein in the osteosarcoma cell lines was higher than that in a normal human osteoblastic cell line, hFOB1.19 $(\mathrm{P}<0.05)$, as shown in Fig. 1.

In order to examine the effect of activin A on osteosarcoma cells, MG63 cells were selected for further study. MG63 cells were transfected with activin A overexpression vector (overexpression group) and activin A siRNA (knockdown group) to enforce activin A expression or inhibit activin A expression in MG63 cells, respectively. MG63 cells without any treatment were used as the blank group. The results from RT-qPCR and western blot analysis demonstrated that activin A exhibited a significant upregulation in the overexpression group and a significant downregulation in the knockdown group compared 
A

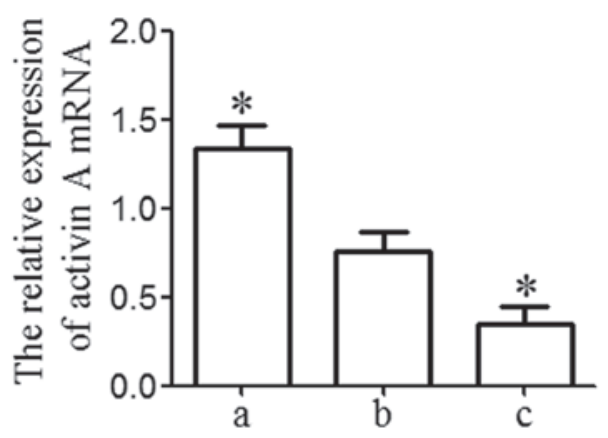

B

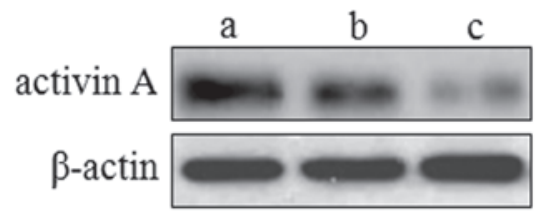

C

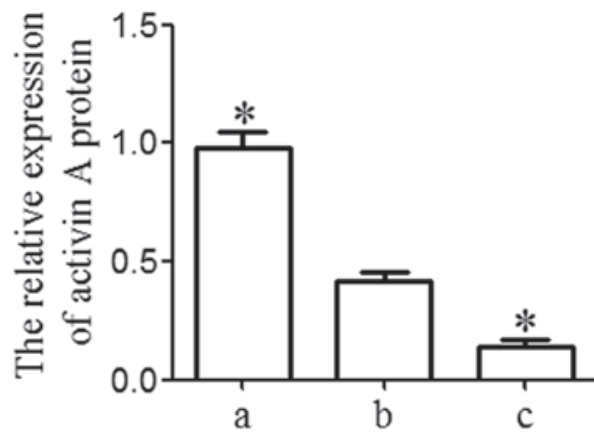

Figure 2. Expression of activin A in MG63 cells. (A) Relative expression of activin A mRNA in MG63 cells. (B) Expression of activin A protein in MG63 cells. (C) Relative expression of activin A protein in MG63 cells. Values are presented as the mean \pm standard deviation of three individual experiments. " $\mathrm{P}<0.05$ compared with $\mathrm{B}$ (a, overexpression group; b, blank group; c, knockdown group).

with that in the blank group $(\mathrm{P}<0.05)$, which indicated that the expression of activin A was effectively enforced or inhibited in MG63 cells (Fig. 2).

Activin A improves MG63 cell viability. The MTT assay demonstrated that MG63 cell viability in the knockdown group was significantly lower than that in the blank group and that MG63 cell viability in the overexpression group was significantly higher than that in the blank group $(\mathrm{P}<0.05$; Fig. 3). These results suggested that activin A may have an important role in the improvement of MG63 cell viability.

Activin A promotes MG63 cell proliferation. Flow cytometric analysis indicated that the number of MG63 cells from the overexpression group in $\mathrm{G}_{0} / \mathrm{G}_{1}$ phase was lower than that from the blank group $(\mathrm{P}<0.05)$ and that the number of MG63 cells from the knockdown group in $\mathrm{G}_{0} / \mathrm{G}_{1}$ phase was higher than that from the blank group $(\mathrm{P}<0.05)$. Furthermore, the results also demonstrated that the number of MG63 cells from the overexpression group in $\mathrm{S}$ and $\mathrm{G}_{2} / \mathrm{M}$ phase was higher than that from the blank group $(\mathrm{P}<0.05)$ and that the number of MG63 cells from the knockdown group in $S$ and $G_{2} / M$ phase was lower than that from the blank group $(\mathrm{P}<0.05)$. The proliferation index $\left[\left(\mathrm{S}+\mathrm{G}_{2} / \mathrm{M}\right) /\left(\mathrm{G}_{0} / \mathrm{G}_{1}+\mathrm{S}+\mathrm{G}_{2} / \mathrm{M}\right)\right]$ was higher in the

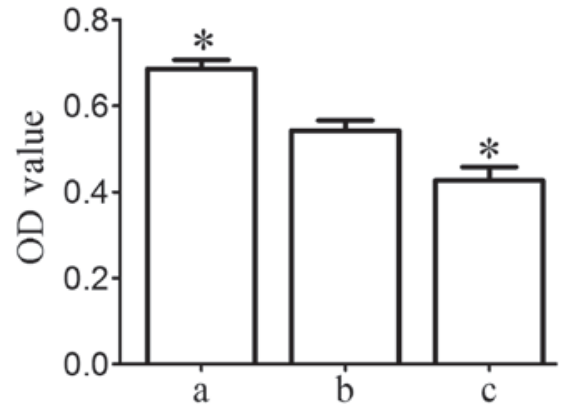

Figure 3. Effect of activin A on MG63 cell viability. Values are presented as the mean \pm standard deviation of three individual experiments. ${ }^{*} \mathrm{P}<0.05$ compared with b. (a, overexpression group; b, blank group; c, knockdown group). OD, optical density.

overexpression group $(\mathrm{P}<0.05)$ and was lower in the knockdown group than that in the blank group $(\mathrm{P}<0.05$; Fig. 4$)$, which led to the conclusion that activin A promotes MG63 cell proliferation.

Activin A improves MG63 cell invasion and migration. Transwell invasion chamber assay demonstrated that the number of MG63 cells invading the Matrigel membrane was significantly lower in the knockdown group and was significantly higher in the overexpression group compared with that in the blank group $(\mathrm{P}<0.05$; Fig. $5 \mathrm{~A}$ and $\mathrm{B})$. These data indicated that activin A may improve MG63-cell invasiveness. Cells in the Transwell inserts and on the lower surface of the inserts were washed into the bottom chamber with medium until no cells remained on the inserts. Cells in the bottom chamber were then incubated with MTT. The OD value was proportional to the number of viable cells. The results indicated that the number of MG63 cells migrated into Transwell inserts on the lower surface of the inserts and into the bottom chamber was significantly higher in the overexpression group and was significantly lower in the knockdown group compared with that in the blank group $(\mathrm{P}<0.05$; Fig. 5C).

Effects of activin A on the expression of cyclin D1, MMP-2 and MMP-9 in MG63 cells. Western blot analysis demonstrated that the expression of cyclin D1, MMP-2 and MMP-9 was significantly upregulated in the overexpression group and significantly downregulated in the knockdown group as compared with that in the blank group $(\mathrm{P}<0.05$; Fig. 6). This suggested that activin $\mathrm{A}$ may be associated with the upregulation of cyclin D1, MMP-2 and MMP-9 in osteosarcoma MG63 cells.

\section{Discussion}

Activin A, belonging to the transforming growth factor- $\beta$ (TGF- $\beta$ ) superfamily, is a multi-functional cytokine. As with all TGF- $\beta$ family members, activin $A$ is a dimeric protein, in this case composed of two activin $\beta$ A subunits (27). Initial investigation into the potential role of activin A focused on inflammatory processes, metabolism, homeostasis, repair, cytoprotection, immune response and endocrine function $(9,10)$. Recent studies have revealed that activin A has an important role in cell proliferation, differentiation, apoptosis and carcinogenesis $(8,11,12)$. However, the roles of activin A signaling in osteosarcoma pathogenesis remain to be elucidated. 

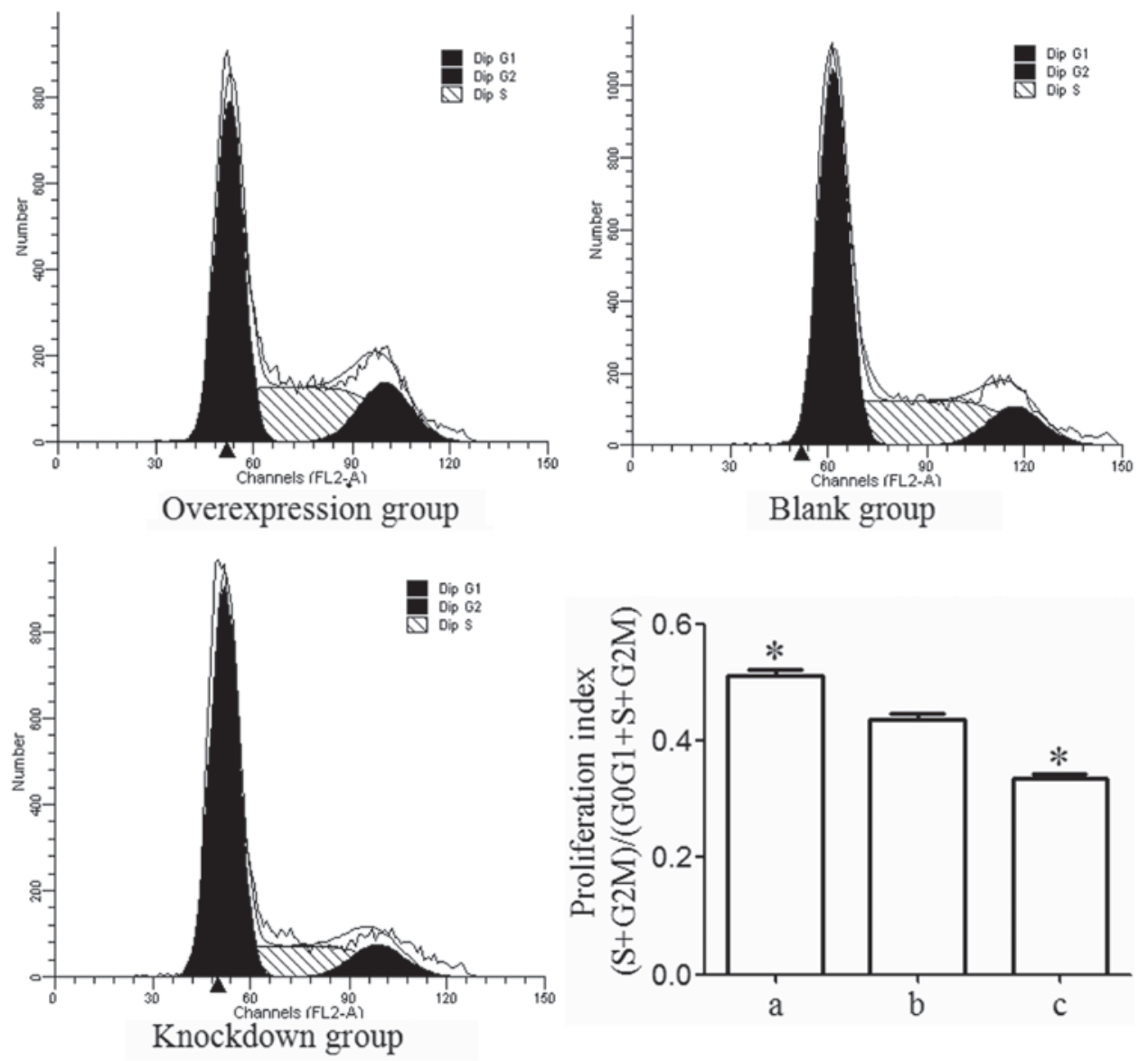

Figure 4. Effect of activin A on MG63 cell cycle. The y-axis represents the proportion of cells in each phase of the cell cycle. ${ }^{*}<<0.05$ compared with b. (a, overexpression group; b, blank group; c, knockdown group). Data were analyzed using one-way analysis of variance. Values are presented as the mean \pm standard deviation and all experiments were repeated three times with three replicates each.

A

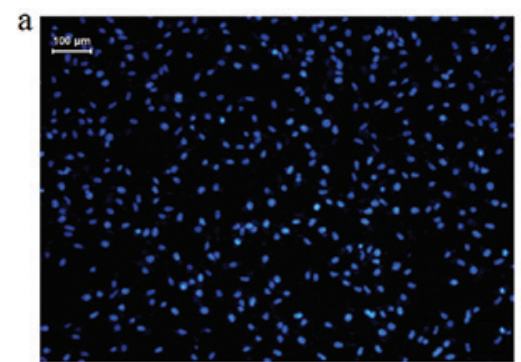

c

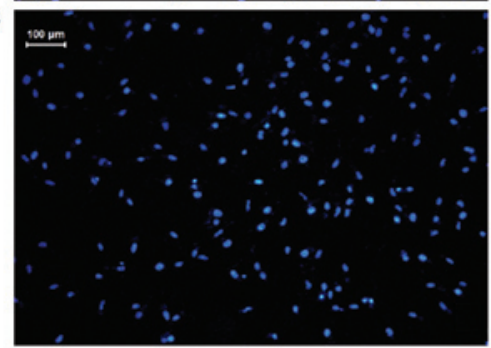

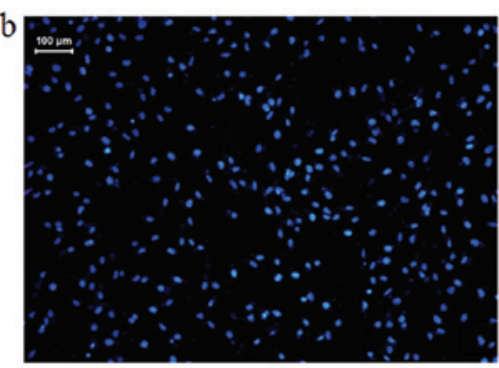
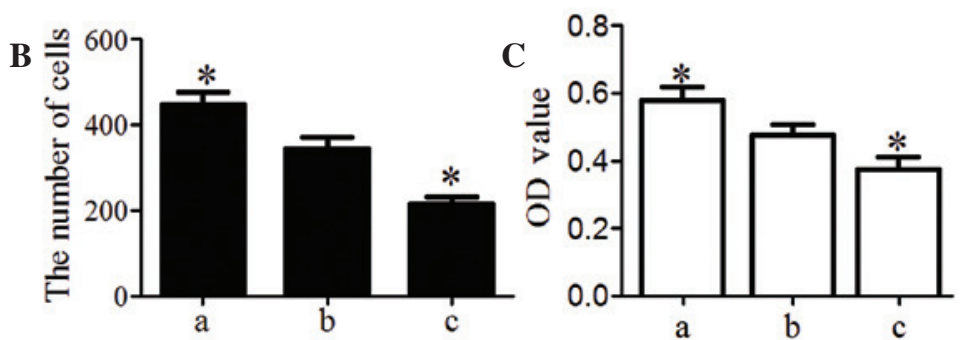

Figure 5. Effect of activin A on MG63 cell invasion ability. (A) Hoechst staining of the MG63 cells that passed through the polycarbonate membrane (a, overexpression group; b, blank group; c, knockdown group). Scale bar, $100 \mu \mathrm{m}$. (B) Quantified number of invaded cells from A. (C) Viability of MG63 cells on the lower surface of the inserts and in the bottom chamber. " $\mathrm{P}<0.05$ compared with $\mathrm{b}$. These data were analyzed using one-way analysis of variance. Values are presented as the mean \pm standard deviation and all experiments were repeated three times with three replicates each.

In the present study, it was identified that activin A was upregulated in the osteosarcoma cell lines (MG63, SaOS-2 and U2OS) compared with the non-cancerous osteoblastic cell line hFOB1.19. This was consistent with the results from a previous study, which demonstrated that activin A was overexpressed in various types of cancer (19). The overexpression of 
A

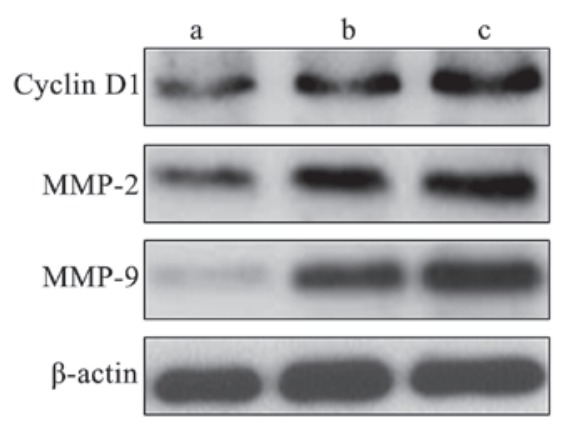

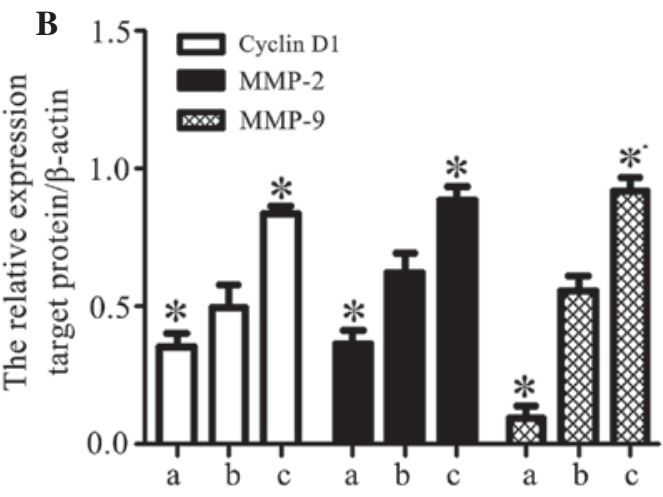

Figure 6. Effects of activin A on the expression of cyclin D1, MMP-2 and MMP-9 in MG63 cells. (A) Cyclin D1, MMP-2 and MMP-9 protein expression in MG63 cells, (B) Relative expression of cyclin D1, MMP-2 and MMP-9 in MG63 cells. (a, overexpression group; b, blank group; c, knockdown group. "P<0.05 compared with b. Data were analyzed using a one-way analysis of variance. Values are presented as the mean \pm standard deviation and all experiments were repeated three times with three replicates each. MMP, matrix metalloproteinase.

activin A may enhance tumor formation and progression (10). Hoda et al (20) demonstrated that activin A exhibited an upregulation in MPM cells. The overexpression of activin A may be responsible for the development of gonadal tumors (21) and was also correlated with positive node stage, poor histological differentiation and perineural invasion (22). Therefore, it was hypothesized that activin A may positively regulate tumor cell proliferation, invasion and migration. To examine the effect of activin A on osteosarcoma cells, MG63 cells were selected and transfected with activin A overexpression vector and activin A siRNA to enforce activin A expression or inhibit activin A expression, respectively. The results of the present study indicated that the expression of activin A was effectively enforced or inhibited in MG63 cells.

The overexpression of activin A contributed to the malignant phenotype of MPM cells (20). Inhibition of activin A in OC3 oral carcinoma cells may attenuate cell proliferation (19). Thus, MG63 cell viability and proliferation were examined by an MTT assay and a flow cytometric assay, respectively. The MTT assay demonstrated that MG63 cell viability in the activin A knockdown group was significantly lower than that in the blank group and that MG63 cell viability in the activin A overexpression group was significantly higher than that in the blank group, which suggested that activin A may have a crucial role in the improvement of MG63 cell viability. The flow cytometric assay indicated that the proliferation index was higher in the activin A overexpression group and was lower in the activin A knockdown group as compared with that in the blank group, which indicated that activin A promotes MG63 cell proliferation. Downregulation of activin A in the conditioned media decreased cell proliferation (28). A previous study also identified that activin A contributed to the malignant phenotype of MPM cells via regulation of cyclin D (20). Silencing of activin A expression by siRNA oligonucleotides led to reduced cyclin D1 expression (20). Therefore, it was hypothesized that activin A improved MG63 cell proliferation, which was possibly via regulation of cyclin D. Therefore, the expression of cyclin D1 was determined by western blotting in the present study. The results demonstrated that cyclin D1 was suppressed in the knockdown group and enhanced in the overexpression group, which may be responsible, at least partially, for the observation that activin A improved MG63 cell proliferation. The results of the present study also suggested that the overexpression of activin A may be a major event in cancer pathogenesis, in part due to improvement of its ability to upregulate cyclin D1, leading to a failure to induce cell cycle arrest. However, further study is required to elucidate the exact mechanism.

The overexpression of activin A was also correlated with migration and invasion. Activin A promotes migration of prostate cancer cells to osteoblasts (29). Yoshinaga et al (22) demonstrated that activin A enhanced migration and invasion of esophageal squamous cell carcinoma cells by enhancing MMP-7 activity (22). Chang et al (19) suggested that suppression of activin A in OC3 oral carcinoma cells may attenuate cell migration and invasiveness. Therefore, the effect of activin A on the invasion and migration ability of MG63 cells in vitro was investigated in the present study. The results revealed that the number of MG63 cells migrated into Transwell inserts on the lower surface of the inserts and into the bottom chamber was significantly higher in the overexpression group and was significantly lower in the knockdown group compared with that in the blank group, which suggested that activin A may improve the abilities of MG63 cell invasion and migration.

Le Bras et al (30) revealed that activin A increases cell invasion through CD44 upregulation following E-cadherin loss and increased CD44 expression in areas of cell invasion associated with MMP-9. In addition, activin A induced in vitro invasion of esophageal squamous cell carcinoma cells, which was accompanied by an increased MMP-2 and MMP-9 in esophageal squamous cell carcinoma cells samples (28). Incorvaia et al (31) demonstrated that activin A, MMP-2 and MMP-9 may be regarded as possible therapeutic targets in the treatment of metastatic bone disease. Therefore, MMP-2 and MMP-9 were examined in the present study. The results revealed that MMP-2 and MMP-9 exhibited significant upregulation in the overexpression group and significant downregulation in the knockdown group compared with that in the blank group, which suggested that activin A may be associated with the upregulation of MMP-2 and MMP-9 in osteosarcoma MG63 cells. A previous study by our group revealed that miR-181a was overexpressed in osteosarcoma cell lines and miR-181a may facilitate proliferation and invasion (32). Neel and Lebrun (12) revealed that activin and TGF- $\beta$ regulated the expression of the miR-181 family to promote cell migration and invasion in breast cancer 
cells (12). Therefore, it was hypothesized that the overexpression of activin A in osteosarcoma may enhance the upregulation of miR-181, facilitating the proliferation and invasion of osteosarcoma cells. This hypothesis requires further investigation.

In conclusion, the results of the present study suggested that activin A may be involved in the enhancement of proliferation, invasion and migration of MG63 cells. However, further study is required to provide a thorough understanding of the function and mechanism of activin $\mathrm{A}$ in osteosarcoma.

\section{Acknowledgements}

The present study was supported by the Natural Science Foundation of Jiangsu Province (grant no. BK20131199) and The 'Six Talent Peaks Program' of the Jiangsu Province of China (grant no. 2011-ws-119).

\section{References}

1. Endo-Munoz L, Evdokiou A and Saunders NA: The role of osteoclasts and tumour-associated macrophages in osteosarcoma metastasis. Biochim Biophys Acta 1826: 434-442, 2012.

2. Poletajew S, Fus L and Wasiutyński A: Current concepts on pathogenesis and biology of metastatic osteosarcoma tumours. Ortop Traumatol Rehabil 13: 537-545, 2011 (In English, Polish).

3. Tanzawa H, Uchiyama S and Sato K: Statistical observation of osteosarcoma of the maxillofacial region in Japan. Analysis of 114 Japanese cases reported between 1930 and 1989. Oral Surg Oral Med Oral Pathol 72: 444-448, 1991.

4. Lee YY, Van Tassel P, Nauert C, Raymond AK and Edeiken J: Craniofacial osteosarcomas: plain film, CT, and MR findings in 46 cases. AJR Am J Roentgenol 150: 1397-1402, 1988.

5. Harting MT and Blakely ML: Management of osteosarcoma pulmonary metastases. Semin Pediatr Surg 15: 25-29, 2006.

6. Liu AN, Zhu ZH, Chang SJ and Hang XS: Twist expression associated with the epithelial-mesenchymal transition in gastric cancer. Mol Cell Biochem 367: 195-203, 2012.

7. Cui J, Wang W, Li Z, Zhang Z, Wu B and Zeng L: Epigenetic changes in osteosarcoma. Bull Cancer 98: E62-E68, 2011.

8. Chen YG, Wang Q, Lin SL, Chang CD, Chuang J and Ying SY: Activin signaling and its role in regulation of cell proliferation, apoptosis, and carcinogenesis. Exp Biol Med (Maywood) 231: 534-544, 2006

9. Sulyok S, Wankell M, Alzheimer C and Werner S: Activin: an important regulator of wound repair, fibrosis, and neuroprotection. Mol Cell Endocrinol 225: 127-132, 2004.

10. Antsiferova $\mathrm{M}$ and Werner S: The bright and the dark sides of activin in wound healing and cancer. J Cell Sci 125 3929-3937, 2012

11. Kypriotou M, Rivero D, Haller S, et al: Activin A inhibits antigen-induced allergy in murine epicutaneous sensitization. Front Immunol 4: 246, 2013.

12. Neel JC and Lebrun JJ: Activin and TGF $\beta$ regulate expression of the microRNA-181 family to promote cell migration and invasion in breast cancer cells. Cell Signal 25: 1556-1566, 2013.

13. Deli A, Kreidl E, Santifaller S, et al: Activins and activin antagonists in hepatocellular carcinoma. World J Gastroenterol 14: 1699-1709, 2008.
14. Matsuo SE, Leoni SG, Colquhoun A and Kimura ET: Transforming growth factor-betal and activin A generate antiproliferative signaling in thyroid cancer cells. J Endocrinol 190: 141-150, 2006.

15. Kaneda H, Arao T, Matsumoto K, et al: Activin A inhibits vascular endothelial cell growth and suppresses tumour angiogenesis in gastric cancer. Br J Cancer 105: 1210-1217, 2011.

16. Burdette JE, Jeruss JS, Kurley SJ, Lee EJ and Woodruff TK: Activin A mediates growth inhibition and cell cycle arrest through Smads in human breast cancer cells. Cancer Res 65: 7968-7975, 2005.

17. Ramachandran A, Marshall ES, Love DR, Baguley BC and Shelling AN: Activin is a potent growth suppressor of epithelial ovarian cancer cells. Cancer Lett 285: 157-165, 2009.

18. Ottley E and Gold E: Insensitivity to the growth inhibitory effects of activin A: an acquired capability in prostate cancer progression. Cytokine Growth Factor Rev 23: 119-125, 2012.

19. Chang KP, Kao HK, Liang Y, et al: Overexpression of activin A in oral squamous cell carcinoma: association with poor prognosis and tumour progression. Ann Surg Oncol 17: 1945-1956, 2010.

20. Hoda MA, Munzker J, Ghanim B, et al: Suppression of activin A signals inhibits growth of malignant pleural mesothelioma cells. Br J Cancer 107: 1978-1986, 2012.

21. Marino FE, Risbridger G and Gold E: The therapeutic potential of blocking the activin signalling pathway. Cytokine Growth Factor Rev 24: 477-484, 2013.

22. Yoshinaga K, Mimori K, Inoue $\mathrm{H}$, et al: Activin A enhances MMP-7 activity via the transcription factor AP-1 in an esophageal squamous cell carcinoma cell line. Int J Oncol 33: 453-459, 2008.

23. Antsiferova M, Huber M, Meyer M, et al: Activin enhances skin tumourigenesis and malignant progression by inducing a pro-tumourigenic immune cell response. Nat Commun 2: 576, 2011.

24. Terpos E, Kastritis E, Christoulas D, et al: Circulating activin A is elevated in patients with advanced multiple myeloma and correlates with extensive bone involvement and inferior survival; no alterations post-lenalidomide and dexamethasone therapy. Ann Oncol 23: 2681-2686, 2012.

25. Livak KJ and Schmittgen TD: Analysis of relative gene expression data using real-time quantitative PCR and the 2(-Delta Delta C(T)) Method. Methods 25: 402-408, 2001.

26. Wang HJ, Ruan HJ,He XJ, et al: MicroRNA-101 is down-regulated in gastric cancer and involved in cell migration and invasion. Eur J Cancer 46: 2295-2303, 2010.

27. Phillips DJ, Jones KL, Clarke IJ, Scheerlinck JP and de Kretser DM: Activin A: from sometime reproductive factor to genuine cytokine. Vet Immunol Immunopathol 108: 23-27, 2005.

28. Sobral LM, Bufalino A, Lopes MA, Graner E, Salo T and Coletta RD: Myofibroblasts in the stroma of oral cancer promote tumorigenesis via secretion of activin A. Oral Oncol 47: 840-846, 2011.

29. Kang HY, Huang HY, Hsieh CY, et al: Activin A enhances prostate cancer cell migration through activation of androgen receptor and is overexpressed in metastatic prostate cancer. J Bone Miner Res 24: 1180-1193, 2009.

30. Le Bras GF, Allison GL, Richards NF, Ansari SS, Washington MK and AndlCD: CD44 upregulation in E-cadherin-negative esophageal cancers results in cell invasion. PloS one 6: e27063, 2011.

31. Incorvaia L, Badalamenti G, Rini G, et al: MMP-2, MMP-9 and activin A blood levels in patients with breast cancer or prostate cancer metastatic to the bone. Anticancer Res 27: 1519-1525, 2007.

32. Jianwei Z, Fan L, Xiancheng L, Enzhong B, Shuai L and Can L: MicroRNA 181a improves proliferation and invasion, suppresses apoptosis of osteosarcoma cell. Tumour Biol 34: 3331-3337, 2013 